\title{
Escala de Motivações para Jogos Online: Estudo de Adaptação à Realidade Brasileira
}

\author{
Valdiney Veloso Gouveia ${ }^{1}$ \\ Tailson Evangelista Mariano \\ Anderson Mesquita do Nascimento \\ Alex Sandro de Moura Grangeiro \\ Universidade Federal da Paraíba, João Pessoa, PB, Brasil \\ Emerson Diógenes de Medeiros \\ Universidade Federal do Piauí, Parnaiba, PI, Brasil
}

\begin{abstract}
Resumo
Este estudo teve como objetivo adaptar ao contexto brasileiro a Escala de Motivações para Jogos Online (EMJO), reunindo evidências de sua validade de construto. Os participantes foram 332 jogadores $(83,8 \%$ do sexo masculino) distribuídos aleatoriamente em dois grupos: $N_{1}$ com 132 jogadores $\left(M_{\text {idade }}=24,8 ; D P\right.$ $=5,80)$ e $N_{2}$ composta por 200 jogadores $\left(M_{\text {idade }}=24,2 ; D P=5,63\right)$. Estes responderam a EMJO e perguntas demográficas. Com o $N_{1}$ foi realizado uma análise dos componentes principais, que mostrou adequada a estrutura com três dimensões com consistência interna aceitável (alfa de Cronbach, $\alpha$ ): realização $(\alpha=$ $0,76)$, imersão $(\alpha=0,78)$ e social $(\alpha=0,72)$. A partir do $N_{2}$, testou-se a estrutura tridimensional por meio de análise fatorial confirmatória. Os indicadores de ajuste deste modelo corroboraram sua adequação (e.g., $\mathrm{CFI}=0,94$, RMSEA $=0,06$ ), tendo os três fatores apresentado consistência interna superior a 0,70. Concluiu-se que esta medida reúne evidências de validade de construto, podendo ser utilizada em estudos futuros.
\end{abstract}

Palavras-chave: Jogos online, escala, validade, precisão.

\section{Online Gaming Motivations Scale: Adaptation Study to the Brazilian Milieu}

\begin{abstract}
This article aimed to adapt and gather evidence of construct validity of the Online Gaming Motivations Scale to the Brazilian context. To this end, counted on the participation of 332 (male 83.8\%) players randomly assigned into two groups: $N_{1}$ with 132 players $\left(M_{\text {age }}=24.8, S D=5.80\right)$, and $N_{2}$ consists of 200 players $\left(M_{\text {age }}=24.2, S D=5.63\right)$. They answered the scale and demographic questions. With the $N_{l}$ was conducted an analysis of the main components, which showed adequate structure with three dimensions with acceptable internal consistency (Cronbach's alpha, $\alpha)$ : achievement $(\alpha=.76)$, immersion $(\alpha=$ $.78)$ and social $(\alpha=.72)$. With $N_{2}$ we tested the three-dimensional structure by a confirmatory factorial analysis. The fit indexes of this model corroborated its suitability (e.g., CFI $=.94$, RMSEA $=.06$ ), with the three factors presented higher internal consistency .70. In conclusion, this measure shows evidence of construct validity, which suggests its use in future research.
\end{abstract}

Keywords: Online games, scale, validity, reliability.

Endereço para correspondência: Universidade Federal da Paraíba, Centro de Ciências Humanas Letras e Artes - Campus I, Departamento de Psicologia, Cidade Universitária, s/n, João Pessoa, PB, Brasil, 58051-900. Fone:

(83) 3216-7856, Fax: (83) 3216-7064. E-mail: vvgouveia@gmail.com 


\section{Escala de Motivaciones para Juegos Online: Estudio de Adaptación a la Realidad Brasileña}

\section{Resumen}

Este estudio tuvo como objetivo la adaptación al contexto brasileño de la Escala de Motivaciones para Juegos Online (EMJO), reuniendo evidencia de su validez de constructo. Participaron 332 jugadores (83.8\% hombres) asignados aleatoriamente en dos grupos: $N_{1}$ con 132 jugadores $\left(M_{\text {edad }}=24.8, S D\right.$ $=5.80)$ y $N_{2}$ con 200 jugadores $\left(M_{\text {edad }}=24.2 ; S D=5.63\right)$. Los participantes respondieron a EMJO y preguntas demográfica. Con la $N_{1}$ se llevó a cabo un análisis de los componentes principales, que ha mostrado la adecuación de uma estrutura con tres dimensiones con consistencia interna aceptable (Alfa de Cronbach, $\alpha)$ : realización $(\alpha=.76)$, inmersión $(\alpha=.78)$ y social $(\alpha=.72)$. Con la $N_{2}$ se analizó la estructura tridimensional mediante un análisis factorial confirmatório. Los indicadores de ajuste de este modelo han corroborado su adecuación (por ejemplo, $\mathrm{CFI}=.94$ y RMSEA $=.06$ ), com los três factor presentando consistencia interna mayor que .70. Se concluye que la medida reune evidencias de validez de constructo pudiendo ser utilizado en estúdios futuros.

Palabras clave: Juegos online, escala, validez, precisión.

O interesse despertado por jogos de computador não é recente. Os primeiros surgiram ainda na segunda metade do século XX, a exemplo do Noughts and Crosses (jogo da velha), desenvolvido em 1952 e do Odyssey, primeiro jogo comercial, lançado em 1972 (Souza, Silva, \& Roazzi, 2010). Posteriormente, com o desenvolvimento de plataformas online, os jogos passaram a possibilitar a interação de múltiplos jogadores unidos em rede (Achab et al., 2011) e, consequentemente, a expansão de jogos em que diversos jogadores interatuam dentro de um mundo virtual com dinâmica própria.

Frente a expansão do número de adeptos de jogos online, tem crescido o número de estudos sobre a dinâmica social e motivacional desses jogadores (Axelsson \& Regan, 2002; Duchenaut \& Moore, 2005; Heckel, 2003; Oliver, 2002; Stansbury, Wheeler, \& Buckingham, 2014; Whang, 2003; Williams et al., 2006; Wing, 2007). Nestes, o que geralmente se verifica é que os aspectos sociais e culturais são os incentivos principais para se jogar, assim como a ligação mantida entre os jogadores (Chen, Duh, Puah, \& Lam, 2006; Cole \& Griffiths, 2007).

Tal interesse parece justificar-se, uma vez que compreender e quantificar as motivações que levam uma pessoa a jogar possibilitará conhecer se os jogadores são motivados diferentemente, e se certas motivações são mais correlacionadas com determinados padrões de uso de jogos e condutas (Kühn, Gleich, Lorenz, Lindenberger, \& Gallinat, 2014). Taxonomia que poderá ser útil tanto para pesquisadores quanto desenvolvedores de jogos, posto que os primeiros poderiam esclarecer se certos tipos de jogadores são mais suscetíveis ao uso problemático dos jogos online, enquanto os desenvolvedores teriam a chance de entender que mecanismos dos jogos atraem mais os jogadores potenciais (Yee, 2006).

\section{Motivações para Jogos Online}

Como pode ser observado, em uma rápida revisão da literatura, algumas taxonomias de tipos de jogadores e suas motivações para jogar foram propostas, sem, no entanto, empregarem métodos estatísticos ou fornecem meios para a sua avaliação quantitativa. Por exemplo, a Bartle's player taxonomy of MUD players (Bartle, 1996), que, embora seja uma taxonomia conhecida para avaliar usuários de jogos online (MUDs), nunca teve testados os pressupostos subjacentes ao modelo.

Embora as limitações práticas, Bartle (1996) defende uma taxonomia estruturada em quatro características que derivam da inter-relação de duas dimensões (ação/interação e orientação mundial/do jogador), na qual a preferência por 
uma dimensão específica do jogo (e.g., realização) suprime as outras dimensões (e.g., socialização, imposição e exploração). Contudo, apesar da construção teórica consistente, não há evidências empíricas de que estes quatro tipos de motivadores são de fato independentes.

Por sua vez, Lazarro (2004) conduziu um estudo observacional entre gêneros que visava conhecer as emoções que mais emergiam de suas experiências de jogos, identificando mais de 30 emoções, entretanto esta investigação não foi desenvolvida utilizando-se de métodos estatísticos, restringindo-se somente a uma descrição de quais emoções eram mais comumente apresentadas em jogos específicos.

Como já mencionado, apesar das contribuições destes autores para o entendimento da motivação para jogos online, estes modelos carecem de evidências de validade e/ou mostram-se muito complexos (Yee, 2006). Com o objetivo de superar essas limitações e desenvolver uma taxonomia mais parcimoniosa Yee, Ducheneaut, e Nelson, 2012 (2012) propuseram a reestruturação da Bartle's player taxonomy of MUD players (Bartle, 1996). Para tal, contou com a participação de 3.000 jogadores, e identificou a existência de dez motivações distribuídas em três grandes fatores relacionados: realização, social e imersão.

O modelo teórico proposto trouxe grandes contribuições, visto que possibilitou a testagem empírica das suas hipóteses e demonstrou-se mais parcimonioso que os anteriormente desenvolvidos. Muito embora os avanços alcançados, limitações, especialmente de ordem metodológica, ainda permaneceram. Sobre esse aspecto, destaca-se que, apesar do modelo identificar três grandes fatores, os 39 itens que compõem a versão original da escala avaliavam os dez componentes adjacentes, sem proporcionar um meio direto de avaliar seus fatores. Ademais, a ausência de estudos fora do contexto amostral com participantes de língua inglesa não asseguram evidências conclusivas sobre a validade da estrutura fatorial em outros contextos culturais (Yee et al., 2012).

\section{Escala de Motivações para Jogos Online}

Frente ao anteriormente descrito, Yee et al. (2012) elaboraram a Escala de Motivações para Jogos Online, que consiste em uma versão reduzida de seu instrumento original (Yee, 2006). A versão ora apresentada é composta por 12 itens distribuídos nos fatores teorizados: realização, social e imersão. O primeiro fator deriva da satisfação em se alcançar objetivos, a exemplo de progredir de nível e acumular recursos; o segundo diz respeito ao interesse do jogador por elementos do ambiente virtual no qual seu personagem está inserido e abrange, por exemplo, a total exploração do mundo e o conhecimento de sua história; e, por último, o terceiro fator expressa o prazer de encontrar e conhecer outros jogadores, conversar e colaborar com eles na conquista de objetivos. Para a efetivação do estudo, tais pesquisadores contaram com uma amostra de 645 asiáticos (Taiwaneses e Chineses), comprovando a estrutura fatorial proposta, explicando conjuntamente $59,3 \%$ da variância total e apresentando alfas de Cronbach de 0,74, 0,77 e 0,75, respectivamente.

Considerando que as pesquisas iniciais a respeito das motivações para se jogar online têm mostrado visões comumente divergentes sobre este construto (Ashinoff, 2014), estabelecer uma taxonomia de motivações como evidências de validade e consistência interna proporcionaria uma ponte teórica e metodológica para entender a relação entre os jogadores e seus comportamentos dentro e fora dos jogos online (Yee et al., 2012). Esta taxonomia, pautada na medida proposta, poderá ajudar na análise das ligações entre dados demográficos, motivações, engajamento, retenção e aprendizagem destes usuários (Zhong, 2011). Nesta direção, reconhecendo a importância de ter instrumentos como deste tipo, pensou-se na realização do presente estudo, cujo objetivo principal foi adaptar a Escala de Motivação para Jogos Online à realidade brasileira, reunindo evidências de seus parâmetros psicométricos. 


\section{Método}

\section{Tradução da Escala de Motivações para Jogos Online}

A fim de contar com uma versão brasileira da Escala de Motivação para Jogos Online, empregou-se a técnica do back-translation. Inicialmente, um psicólogo bilíngue traduziu a versão original para o português, versão que foi posteriormente retraduzida para o inglês por um segundo psicólogo também com fluência nos dois idiomas. Ao fim desse processo, um terceiro tradutor, também fluente nos dois idiomas, ficou responsável por comparar a versão retraduzida com a original do instrumento.

Unicamente houve modificações de forma, produzindo-se a versão final desta medida; assegurada a equivalência de conteúdo, procedeu-se a etapa de validação semântica da escala, aplicando-a a dez jogadores. Esta etapa buscou constatar a inteligibilidade dos itens, a compreensão de expressões típicas de jogos online e a adequação da escala de resposta. Não foi identificada qualquer necessidade de modificar substancialmente qualquer item da versão em português, que foi posteriormente empregada no estudo. Esta versão está disponível sob solicitação a um dos autores deste artigo.

\section{Participantes}

Para a efetivação desta pesquisa, contouse com a participação de 332 jogadores de diferentes estados do Brasil. Com o intuito de verificar a validade cruzada e a estabilidade fatorial da medida, a amostra total foi aleatoriamente dividida em dois grupos (Laros, 2005; Schumacker \& Lomax, 2004), descritos a seguir: $N_{1}=$ formado por 132 jogadores com idades variando entre 15 e 53 anos $(M=24,8$; $D P=5,80$ ), sendo a maioria do sexo masculino $(83,2 \%)$, solteira $(72,0 \%)$, sem religião $(44,7 \%)$ e de classe média $(28,0 \%)$ e residentes no estado de São Paulo (18,2\%); e $N_{2}=$ composta por 200 jogadores, cujas idades variaram entre 14 e $44 \operatorname{anos}(M=24,2 ; D P=5,63)$, sendo a maioria do sexo masculino $(85,9 \%)$ e solteira $(79,5 \%)$, sem religião $(51,5 \%)$ e de classe média $(28,5 \%)$ e residentes no estado de São Paulo
$(20,0 \%)$. Destaca-se que ambas as amostras foram de conveniência (não probabilística) e que o tamanho amostral foi estabelecido conforme recomendações presentes na literatura da área, a qual pressupõe um mínimo de 10 respondentes por item avaliado, para o emprego de análise fatorial exploratória (Pasquali, 2010) e um mínimo de 200 observações, para a realização da análise fatorial confirmatória (Kelloway, 2008).

\section{Instrumentos}

A versão digital do questionário que os participantes responderam constava de duas partes principais, a saber: Questões Sócio-Demográficas em formato fechado (e.g. sexo, idade, estado civil, religião e classe econômica autopercebida) e a Escala de Motivação para Jogos Online.

Escala de Motivação para Jogos Online. Originalmente elaborada em língua inglesa por Yee et al. (2012), esta escala é composta por 12 itens distribuídos em três dimensões, relativas às motivações para jogar: realização (e.g., Melhorar o seu personagem o máximo possivel), social (e.g., Conversar com outros jogadores) e imersão (e.g., Explorar o mundo com o objetivo de conhecê-lo). Os participantes são convidados a indicar o grau de importância que cada comportamento tem quando se está jogando jogos online, utilizando escala de resposta do tipo Likert, de cinco pontos, variando de 1 (Não importante) a 5 (Muito importante).

\section{Procedimento}

A coleta de dados foi realizada com uma versão digital da escala, similar ao questionário impresso, a qual foi disponibilizada de forma online por meio da plataforma LimeSurvey, hospedada na página web do grupo de pesquisa dos autores. Dada a disponibilidade de contar com a versão online do questionário, os contatos e a solicitação de participação ocorreram por meio de fóruns dedicados a jogadores de jogos online. Nestes espaços, foram criados tópicos públicos nos quais foi disponibilizada uma breve descrição da pesquisa e o endereço de acesso para aqueles que optassem por participar do estudo. Em consonância com a Resolução do Conselho Nacional de Saúde (CNS) no 466/12, todos os 
princípios éticos foram respeitados, garantindo aos respondentes o anonimato de suas respostas, sua participação voluntária e a possibilidade de declínio do preenchimento em qualquer momento sem prejuízo, tendo sido aprovado pelo Comitê de Ética em Pesquisa da Universidade Federal da Paraíba (Parecer $\mathrm{N}^{\circ}$ 1.159.428). Para documentar o consentimento livre e esclarecido, antes de iniciar o preenchimento do questionário, foi solicitado a cada participante que, caso concordasse com o Termo de Consentimento Livre e Esclarecido, assinalasse a opção "Aceito Participar da Pesquisa" atestando a sua anuência e a compreensão dos objetivos da pesquisa. Em média, as pessoas levaram cerca de 5 minutos para concluir sua participação no estudo.

\section{Análise dos Dados}

Os dados foram inicialmente analisados com o pacote estatístico PASW (Predictive Analytics Software; versão 18). Por meio deste, foram calculadas estatísticas descritivas (medidas de tendência central, dispersão e distribuição de frequência), poder discriminativo dos itens ( $t$ de Student), análise dos componentes principais (CP) e alfa de Cronbach (consistência interna). Posteriormente, por meio do pacote estatístico AMOS (Analysis of Moment Structures; versão 18), buscou-se conhecer a adequação da solução fatorial observada na análise de CP. No caso, considerou-se a matriz de covariância, tendo sido adotado o estimador ML (Maximum Likelihood). Os seguintes indicadores de ajuste foram considerados (Byrne, 2013; Garson, 2003; Tabachnick \& Fidell, 2013):

Razão $\chi^{2} / g$.l. (qui-quadrado / grau de liberdade), admitindo-se um valore de até 5 como indicativo de ajustamento adequado;

Goodness-of-Fit Index (GFI), cujos valores variam de 0 a 1 , com aqueles próximos a 0,90 indicando um ajustamento satisfatório do modelo teórico na explicação da matriz de variância-covariância;

Comparative Fit Index (CFI), índice comparativo, adicional, de ajuste ao modelo, com valores mais próximos de 1 indicando melhor ajuste com 0,90 sendo a referência para aceitar o modelo; e
Root-Mean-Square Error of Approximation (RMSEA), indicando ajuste entre o modelo teórico esperado e os dados empíricos obtidos, sendo que quanto mais próximo de zero, melhor, admitindo-se valores de até 0,10 (ponto de corte de 0,08 ). Adicionalmente, visando ampliar as evidências acerca da validade de construto da escala (Fornell \& Larcker, 1981; Hair, Black, Babin, Anderson, \& Tathan, 2009), foram calculados os coeficientes de confiabilidade composta (CC) dos fatores da medida, tomando em conta os resultantes das análises prévias.

\section{Resultados}

\section{Análises dos Itens e Estrutura Fatorial da Escala}

Nesta oportunidade foram considerados os participantes do primeiro grupo amostral $\left(N_{1}\right)$, procurando, primeiramente, conhecer o poder discriminativo dos 12 itens que compõem a escala. No caso, teve-se em conta o critério da mediana empírica da pontuação total (soma das respostas de cada participante em todos os itens; $M d=45$ ) para definir os grupos-critério (Pasquali, 2010). Posteriormente, por meio de um teste $t$, buscou-se verificar em que medida cada um dos itens conseguiu discriminar satisfatoriamente os participantes do grupo inferior e superior. Em suma, como observado, todos os itens foram capazes de diferenciar os participantes dos dois grupos na direção esperada $(t>3, p<0,001)$. Os resultados são, pormenorizadamente, apresentados na Tabela 1 a seguir:

Com o fim de checar a adequação dos dados à realização de análise fatorial exploratória, observaram-se seu indicador de Kaiser-MeyerOlkin $(\mathrm{KMO}=0,70)$ e Teste de Esfericidade de Bartle $\left[X^{2}(66)=564,17, p<0,001\right]$. Estes claramente apoiaram que se levasse a cabo este tipo de análise (Tabachnick \& Fidell, 2013), optando-se pelo método de componentes principais $(\mathrm{CP})$ com rotação promax, sem fixar o número de fatores a extrair. Foi possível identificar três componentes com valores próprios (eigenvalues) superiores a 1 (Critério de Kaiser), os quais explicaram conjuntamente $61,7 \%$ da variância total. Conforme pode ser visto 
Tabela 1

Poder Discriminativo dos Itens da de Motivações para Jogos Online

\begin{tabular}{|c|c|c|c|c|c|c|c|}
\hline \multirow{3}{*}{ Itens } & \multicolumn{4}{|c|}{ Grupo Critério } & \multirow{2}{*}{\multicolumn{3}{|c|}{ Contraste }} \\
\hline & \multicolumn{2}{|c|}{ Superior } & \multicolumn{2}{|c|}{ Superior } & & & \\
\hline & $M$ & $D P$ & $M$ & $D P$ & $t$ & $g l$ & $p$ \\
\hline 1 & 3,40 & 1,06 & 4,06 & 0,86 & 3,84 & 117 & $<0,001 *$ \\
\hline 2 & 2,76 & 1,14 & 4,03 & 1,02 & 6,66 & 122 & $<0,001 *$ \\
\hline 3 & 3,34 & 1,03 & 4,06 & 0,84 & 4,31 & 117 & $<0,001 *$ \\
\hline 4 & 3,90 & 1,03 & 4,47 & 0,70 & 3,68 & 128 & $<0,001 *$ \\
\hline 5 & 3,48 & 1,22 & 4,41 & 0,86 & 4,94 & 108 & $<0,001 *$ \\
\hline 6 & 3,34 & 1,20 & 4,44 & 0,69 & 6,32 & 96 & $<0,001 *$ \\
\hline 7 & 3,82 & 1,09 & 4,62 & 0,57 & 5,11 & 90 & $<0,001 *$ \\
\hline 8 & 2,84 & 1,20 & 4,07 & 1,06 & 6,19 & 128 & $<0,001 *$ \\
\hline 9 & 3,68 & 1,25 & 4,63 & 0,57 & 5,50 & 83 & $<0,001 *$ \\
\hline 10 & 3,81 & 1,09 & 4,69 & 0,55 & 5,71 & 88 & $<0,001 *$ \\
\hline 11 & 4,06 & 1,05 & 4,84 & 0,40 & 5,42 & 77 & $<0,001 *$ \\
\hline 12 & 3,19 & 1,35 & 4,07 & 1,11 & 4,03 & 118 & $<0,001 *$ \\
\hline
\end{tabular}

Nota. * Item discriminativo.

na Figura 1, a legitimidade da retenção de três componentes foi corroborada por dois critérios mais robustos: Cattell (distribuição gráfica dos valores próprios) e Horn (análise paralela, isto é, simulação de valores próprios a partir de matrizes de dados aleatórios, reproduzindo os parâmetros do banco original em termos de itens e participantes).

Com base nos resultados previamente descritos, coerente com a estrutura observada para a escala no estudo em que ela foi proposta, decidiu-se fixar a extração de três componentes, realizando-se nova análise de $\mathrm{CP}$. Os achados a respeito podem ser verificados na Tabela 2, sendo destacados os itens cujas cargas fatoriais foram iguais ou superiores a $|0,30|$; estes foram assumidos como representando o fator correspondente.

O primeiro componente foi denominado como realização. Este reuniu quatro itens com saturações variando entre 0,55 (Item 12. Competir com outros jogadores) e 0,88 (Item 09.
Tornar-se poderoso), explicando $28,1 \%$ da variância total. Sua consistência interna, avaliada por meio do alfa de Cronbach $(\alpha)$ foi de 0,76 .

O segundo componente foi nomeado como imersão. Ele reuniu quatro itens com saturações variando entre 0,70 (Item 06. Sentir-se parte do mundo [no jogo]) e 0,85 (Item 07. Explorar o mundo com o objetivo de conhece-lo), tendo explicado $18,7 \%$ da variância total e apresentado (alfa de Cronbach, $\alpha$ ) de 0,78 .

Por fim, o terceiro componente, denominado social, agregou os quatro itens restantes, os quais apresentaram saturações variando entre 0,47 (Item 04. Manter contato com os meus amigos) e 0,85 (Item 03. Participar de grupos com outros jogadores). Este componente explicou $15,0 \%$ da variância total, apresentando coeficiente aceitável de consistência interna ( $\alpha$ $=0,72$ ).

Em resumo, esses achados sugerem evidências de validade fatorial e consistência interna da Escala de Motivação para Jogos Online. Con- 


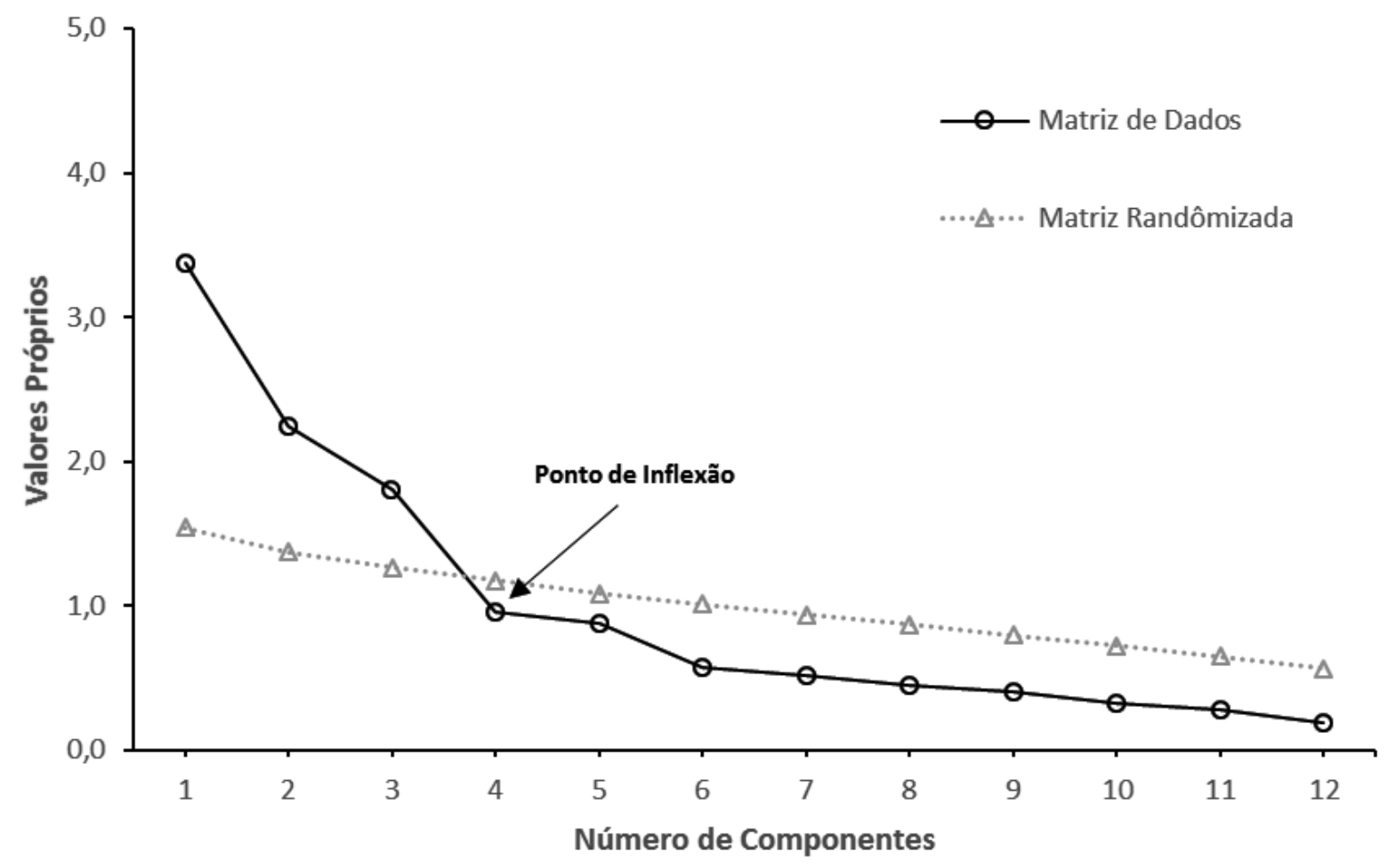

Figura 1. Diagrama de Declividade (scree plot) da Medida de Motivações para Jogos Online.

tudo, reconhecendo o caráter essencialmente exploratório das análises realizadas, decidiu-se testar a adequação de sua estrutura formada por três componentes, efetuando uma análise fatorial confirmatória (CFA). Isso permitirá conhecer em que medida os resultados prévios são replicáveis em um grupo diferente de participantes, o que se leva a cabo a seguir.

\section{Comprovando a Estrutura Fatorial da Medida de Motivação para Jogos Online}

Nesta oportunidade se teve em conta o segundo grupo amostral deste estudo $\left(N_{2}\right)$. Concretamente, efetuou-se uma CFA considerando a matriz de covariância como entrada e empregando o estimador ML (Maximum Likelihood). Procurou-se definir a estrutura de acordo com a pertença dos itens por fator, de acordo com a Tabela 2. Os indicadores de ajuste do modelo correspondente revelaram uma solução satisfatória: $\chi^{2}(51)=99,80, p<0,001$, $\chi^{2} / g l=1,95, \mathrm{GFI}=0,92, \mathrm{CFI}=0,94$ e RMSEA $=0,06($ IC $90 \%=0,049-0,089)$. Todos os itens apresentaram saturações (pesos fatoriais, $\lambda$ ) estatisticamente diferentes de zero $(\lambda \neq 0 ; z$ $>1,96, p<0,05)$. A estrutura correspondente pode ser observada na Figura 2 a seguir.

Portanto, comprovada a pertinência e o ajuste aceitável do modelo, procurou-se reunir evidências complementares de sua validade de construto e consistência interna. A semelhança do que se observou para o grupo de participantes previamente considerado $\left(N_{1}\right)$, neste os coeficientes de consistência interna foram igualmente satisfatórios para os três componentes da medida, isto é, realização ( $\alpha$ $=0,79)$, imersão $(\alpha=0,73)$ e social $(\alpha=0,80)$. Corroboram esse parâmetro os coeficientes de confiabilidade composta ( $C C=0,81,0,75$ e 0,83 , respectivamente).

\section{Discussão}

No geral, tomando-se em conta os resultados, parece evidente que as motivações para jogos online podem ser parcimoniosamente agrupadas em três componentes (realização, imersão e social), corroborando a estrutura observada quando da elaboração desta medida 
Tabela 2

Estrutura Fatorial da Medida de Motivações para Jogos Online

\begin{tabular}{lcccc}
\hline & \multicolumn{3}{c}{ Componente } & \\
\cline { 2 - 4 } Conteúdo & I & II & III & \\
\hline Tornar-se poderoso. & $\mathbf{0 , 8 8}$ & $-0,02$ & 0,07 & 0,77 \\
Adquirir itens raros. & $\mathbf{0 , 8 6}$ & $-0,08$ & $-0,13$ & 0,78 \\
Melhorar o seu personagem o máximo possível. & $\mathbf{0 , 7 8}$ & $-0,03$ & $-0,15$ & 0,67 \\
Competir com outros jogadores. & $\mathbf{0 , 5 5}$ & 0,10 & 0,08 & 0,32 \\
Explorar o mundo com o objetivo de conhecê-lo. & $-0,04$ & $\mathbf{0 , 8 5}$ & 0,06 & 0,71 \\
Criar uma história para o seu personagem. & 0,18 & $\mathbf{0 , 7 8}$ & 0,15 & 0,65 \\
Aprender sobre as histórias e a cultura do mundo. & $-0,18$ & $\mathbf{0 , 7 5}$ & $-0,10$ & 0,60 \\
Sentir-se parte do mundo (no jogo). & 0,11 & $\mathbf{0 , 7 0}$ & $-0,22$ & 0,62 \\
Participar de grupos com outros jogadores. & 0,06 & $-0,11$ & $\mathbf{- 0 , 8 5}$ & 0,73 \\
Conversar com outros jogadores. & $-0,11$ & $-0,05$ & $\mathbf{- 0 , 8 5}$ & 0,69 \\
Fazer parte de uma guilda ou clã. & 0,23 & 0,04 & $\mathbf{- 0 , 7 0}$ & 0,61 \\
Manter contato com os seus amigos (as). & $-0,03$ & 0,19 & $\mathbf{- 0 , 4 7}$ & 0,29 \\
Valor Próprio & 3,36 & 2,24 & 1,80 & \\
\% Variância Explicada & 28,1 & 18,7 & 15,0 & \\
Alfa Cronbach & 0,76 & 0,78 & 0,72 & \\
\hline
\end{tabular}

Nota. $h^{2}$ - Comunalidade. Itens destacados em negrito possuem cargas fatoriais iguais ou superiores a $|0,30|$.

(Yee et al., 2012). Tal resultado também reforça o pressuposto da taxonomia proposta por Yee (2006) e demonstra a sua estabilidade, mesmo considerando um contexto cultural distinto daquele onde foi desenvolvida.

Sobre tal aspecto, assim como em sua formulação original (Yee et al., 2012), o primeiro componente, denominado realização, reuniu itens que avaliam a motivação do jogador para obter poder, seja por meio de acessórios raros ou tornando seu personagem o mais forte possível com o intuito de obter o melhor desempenho ao competir com outros jogadores. A existência desse componente também foi observada por Bartle (1996), no entanto, ao contrário de sua formulação teórica, não há evidências empíricas que a preferência por esta dimensão suprima a preferência pelas demais, como propunha este autor.
No que se refere aos outros componentes, o segundo, imersão, diz respeito a motivação do jogador em sentir-se parte do jogo, tendo a oportunidade de customizar um personagem que construa uma história própria e que possibilite aprender sobre as histórias e a cultura do jogo. Por fim, o último componente, social, tem como base a motivação do jogador para desenvolvimento e manutenção de relações interpessoais com outros jogadores, inclusive formando grupo com eles. A existência deste último fator parece reforçar a premissa de que os aspectos sociais dos jogos online são importantes incentivos para se jogar, assim como a ligação mantida entre os jogadores (Chen et al., 2006; Cole \& Griffiths, 2007).

Quanto a esses achados, ressalta-se que a estrutura indicada emergiu livremente dos dados. O que reforça a adequação psicométrica da 


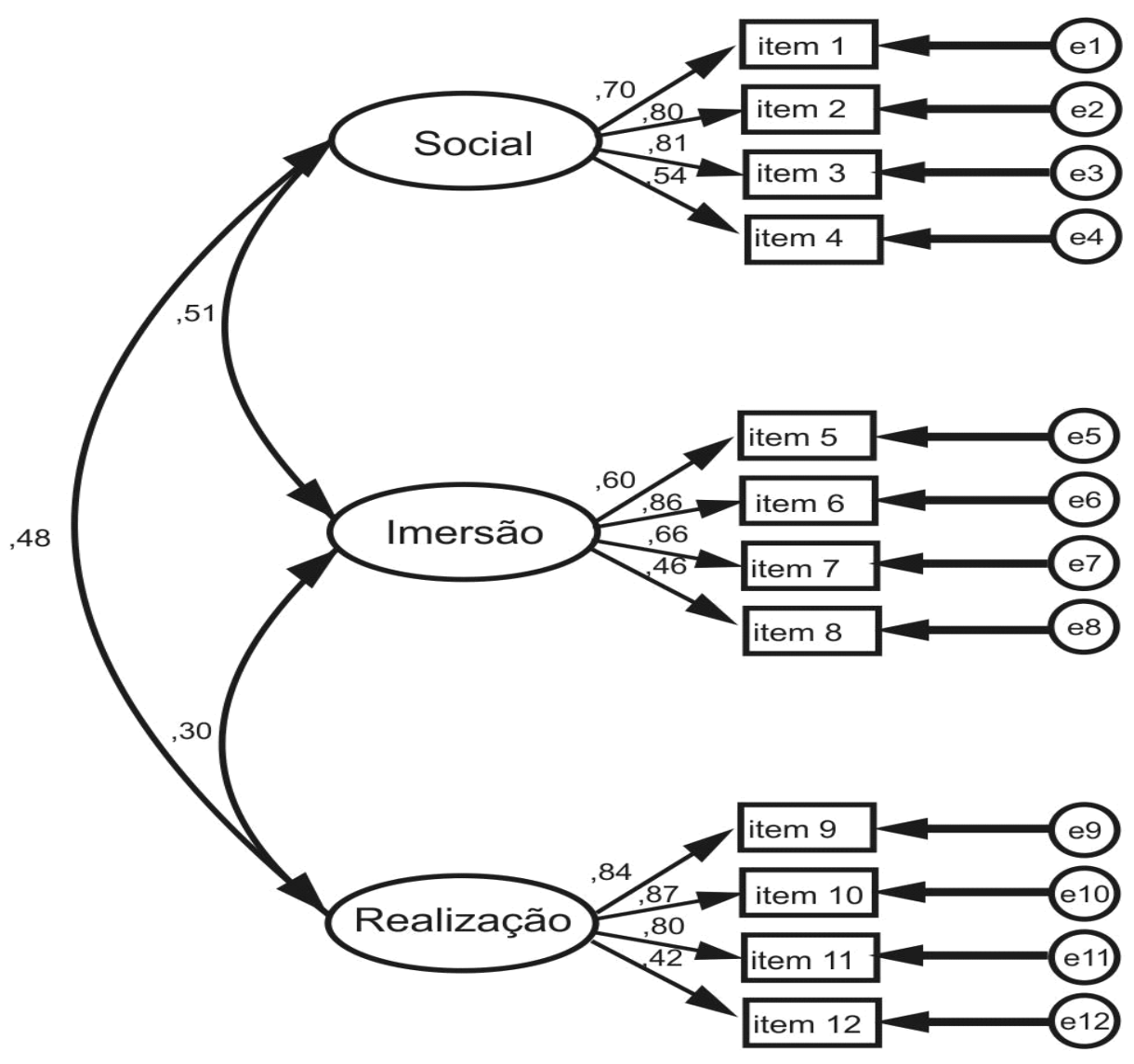

Figura 2. Estrutura Fatorial da Escala de Motivações para Jogos Online.

medida. Ademais foram observados os indicadores de consistência interna (alfa de Cronbach, $\alpha$ ) e confiabilidade composta (CC) superiores aos recomendados na literatura $(0,70$; Hair et al., 2009; Pasquali, 2010). Portanto, parece evidente que se trata de um instrumento que mede o construto de interesse de forma satisfatória.

Embora evidentes as contribuições desta pesquisa, apontam-se algumas de suas limitações potenciais. Sobre esse aspecto, ainda que se tenha contribuído no sentido de replicar a estrutura motivacional para jogos online, conforma proposta por Yee et al. (2012) em uma cultura diferente da qual foi desenvolvida, reconhece-se que os achados ora disponibilizados são resultantes de uma amostra de conveniência, não sendo representativa da população geral, o que limita a generalização dos resultados. A propósito da amostra, há que assinalar que o estudo reuniu mais jogadores do sexo masculino, sendo que o sexo poderá ser uma variável relevante neste contexto, cabendo testar se a medida em pauta apresenta evidências de invariância fatorial com respeito ao sexo (Yee, 2006).

Como tal, será interessante replicá-lo em amostra mais equitativa em relação ao sexo dos participantes, checando eventuais diferenças nas motivações entre homens e mulheres em contexto brasileiro. Sugere-se, ainda, considerar parâmetros psicométricos adicionais desta medida, a exemplo de sua estabilidade temporal (precisão teste-reteste) e validade convergente, atentando-se a relação entre motivação para jogos online e construtos correlatos, como traços de personalidade (Bargh, McKenna, \& Fitzsimons, 2002), comportamentos sociais (Greitemeyer \& Mügge, 2014) e compulsividade (Medeiros et al., no prelo).

Finalmente, mesmo que o presente estudo não tenha cunho eminentemente prático, a medida ora adaptada favorece que seja empregada para entender o perfil do jogador ao iniciar um jogo 
online, o que abre a possibilidade de adaptar a experiência do jogo para melhor atender às suas motivações (Riecken, 2000). Também poderá servir, no contexto clínico, para conhecer que aspectos podem ser determinantes da conduta de se envolver com jogos virtuais, permitindo identificar problemas potenciais que demandarão a atenção, favorecendo que tais jogos não se tornem um problema (por exemplo, a renúncia aos laços interpessoais), mas uma alternativa saudável de entretenimento.

Deste modo, justifica-se o seu uso em contexto de pesquisa e, provavelmente, intervenções sociais. Neste ponto, tendo-se em conta que os jogos virtuais são grandes plataformas de entretenimento, o emprego da EMJO possibilitará auxiliar na compreensão de fenômenos sociais complexos que estão surgindo nos mundos virtuais todos os dias, e que, em sua maioria, são inexplorados, a exemplo das motivações que levam algumas pessoas a preferirem jogos violentos (Kasumovic, Blake, Dixson, \& Denson, 2015; Yee, 2006).

\section{Referências}

Achab, S., Nicolier, M., Mauny, F., Monnin, J., Trojak, B., Vandel, P., \& Haffen, E. (2011). Massively multiplayer online role-playing games: Comparing characteristics of addict vs non-addict online recruited gamers in a French adult population. BMC Psychiatry, 11, 144. doi:10.1186/1471-244X-11-144

Ashinoff, B. K. (2014). The potential of video games as a pedagogical tool. Frontiers in Psychology, 5, 1109. doi:10.3389/fpsyg.2014.01109

Axelsson, A., \& Regan, T. (2002). How belonging to an online group affects social behavior $-A$ case study of Asheron's call (Technical Report MSR-TR-2002-07). Redmond, WA: Microsoft Corporation One Microsoft Way.

Bargh, J., McKenna, K., \& Fitzsimons, G. (2002). Can you see the real me? Activation and expression of the "true self"on the internet. Journal of Social Issues, 58, 33-48. doi:10.1111/15404560.00247

Bartle, R. (1996). Hearts, clubs, diamonds, spades: Players who suit MUDs. Journal of $M U D$ Research, 1(1), 19.
Byrne, B. M. (2013). Structural equation modeling with AMOS: Basic concepts, applications and programming. Mahwah, NJ: Erlbaum.

Chen, V. H., Duh, H. B., Puah, P. S. K., \& Lam, D. Z. (2006). Enjoyment or engagement? Role of social interaction in playing massively multiplayer online role-playing games (MMORPGS). In Entertainment computing - ICEC 2006 (pp. 262-267). Berlin, Germany: Springer. doi:10.1007/11872320 31

Cole, H., \& Griffiths, M. D. (2007). Social interactions in massively multiplayer online role-playing gamers. CyberPsychology \& Behavior, 10, 575583. doi:10.1089/cpb.2007.9988

Conselho Nacional de Saúde. (2012). Resolução CNS $n^{\circ}$ 466/12. Recuperado em http://conselho.saude.gov.br/resolucoes/2012/Reso466.pdf

Duchenaut, N., \& Moore, R. J. (2005). More than just 'XP': Learning social skills in massively multiplayer online games. Interactive Technology \& Smart Education, 2, 89-100. doi:10.1108/17415650580000035

Fornell, C., \& Larcker, D. F. (1981). Evaluating structural equation models with unobservable variables and measurement error. Journal of Marketing Research, 18, 39-50. doi: $10.2307 / 3150980$

Garson, G. D. (2003, June 24). PA 765 Statnotes: An online textbook. Retrieved from http://www2. chass.ncsu.edu/garson/pa765/statnote.htm

Greitemeyer, T., \& Mügge, D. (2014). Video games do affect social outcomes: A meta-analytic review of the effects of violent and prosocial video dame. Personality and Social Psychology Bulletin, 40, 578-589. doi:10.1177/0146167213520459

Hair, F. J., Black, W. C., Babin, B., Anderson, R. E., \& Tathan, R. L. (2009). Análise multivariada de dados (6. ed.). Porto Alegre, RS: Bookman.

Heckel, H. L. (2003). Online social interaction: The case of Everquest (Master's thesis, Faculty of George Mason University, Fairfax, VA, USA).

Kasumovic, M., Blake, K., Dixson, B., \& Denson, T. (2015). Why do people play violent video games? Demographic, status-related, and mating-related correlates in men and women. Personality and Individual Differences, 86, 204-211. doi:10.1016/j.paid.2015.06.018 
Kelloway, E. K. (2008). Using LISREL for structural equation modeling: A researcher's guide. Thousand Oaks, CA: Sage.

Kühn, S., Gleich, T., Lorenz, R. C., Lindenberger, U., \& Gallinat, J. (2014). Playing Super Mario induces structural brain plasticity: Gray matter changes resulting from training with a commercial video game. Molecular Psychiatry, 19(2), $265-271$

Laros, J. A. (2005). O uso de análise fatorial: Algumas diretrizes para pesquisadores. In L. Pasquali (Ed.), Análise fatorial para pesquisadores (pp. 163-184). Brasília, DF: Laboratório de Pesquisa e Medida, Universidade de Brasília.

Lazarro, N. (2004). Why we play games: Four keys to more emotion without story. $G D C$.

Medeiros, E. D., Sales, H. F. S., Lopes, B. J., Mariano, T. E., Nascimento, A. M., \& Silva, P. G. N. (no prelo). Adaptação da escala de uso compulsivo da Internet (CIUS) para o Brasil. Psicologia Argumento (PUCPR. Impresso).

Oliver, J. H. (2002). The similar eye: Proxy life and public space in the MMORPG. In Proceedings of the 2002 Computer Games and Digital Cultures Conference (pp. 171-184). Tampere, Finland: Tampere University Press.

Pasquali, L. (2010). Instrumentação psicológica: Fundamentos e práticas. Porto Alegre, RS: Artmed.

Riecken, D. (2000). Personalized views of personalization. Communications of the ACM, 43(8), 26-28.

Schumacker, R. E., \& Lomax, R. G. (2004). A beginner's guide to structural equation modeling. New York: Psychology Press.

Souza, B. C., Silva, L. X. D. L., \& Roazzi, A. (2010). MMORPGS and cognitive performance: A study with 1280 Brazilian high school students. Computers in Human Behavior, 26(6), 15641573.

Stansbury, J. A., Wheeler, E. A., \& Buckingham, J. T. (2014). Can Wii engage college-level learners? Use of commercial off-the-shelf gaming in an introductory statistics course. Computers in the Schools, 31(1-2), 103-115.
Tabachnick, B. G., \& Fidell, L. S. (2013). Using Multivariate Statistics ( $6^{\text {th }}$ ed.). Boston, MA: Pearson.

Whang, L. S. (2003). Online game dynamics in Korean society: Experiences and lifestyles in the online game world. Korea Journal, 43, 7-34.

Williams, D., Ducheneaut, N., Xiong, L., Zhang, Y., Yee, N., \& Nickell, E. (2006). From tree house to barracks: The social life of guilds in world of warcraft. Games and Cultures, 1, 338-361. doi:10.1177/1555412006292616

Wing, J. S. L. (2007, June 24). Interpersonal relationships: From aggression and hatred to altruism and love - How should one perceive another in a computer game. Retrieved from http:/www.cs.mcgill.ca/ jlionw/COMP763/ report.pdf

Yee, N. (2006). Motivations for play in online games. Journal of CyberPsychology and Behavior, 9(6), 772-775. doi:10.1089/cpb.2006.9.772

Yee, N., Ducheneaut, N., \& Nelson, L. (2012). Online gaming motivations scale: Development and validation. In Proceedings of the SIGCHI Conference on Human Factors in Computing Systems, Austin, TX. doi:10.1145/2207676.2208681

Zhong, Z. J. (2011). The effects of collective MMORPG (Massively Multiplayer Online Role-Playing Games) play on gamers' online and offline social capital. Computer in Human Behavior, 27, 2352-2363. doi:10.1016/j. chb.2011.07.014
Recebido: $18 / 09 / 2015$

$1^{a}$ revisão: 10/01/2016

Aceite final: 26/01/2016 